\title{
Fuzzy Inference Using Bee Colony Optimization for Redeem Routing inMANET
}

\author{
Dr Geetha . N \\ \{*sng.mca@psgtech.ac.in $\}$ \\ Assistant Professor (Selection Grade),Department of Computer Applications, PSG College of \\ Technology, Tamilnadu, Coimbatore-641004, India
}

\begin{abstract}
Mobile ad-hoc network (MANET), are designed especially for mobile nodes without any infrastructure. Because of its mobility nature, route failure occurs frequently resulting in data loss and overheads. In order to overcome above drawback, the routing has to be done in accordance with mobility character of the network. In this paperFuzzy Inference Using Bee Colony Optimization for Redeem Routing in MANETis proposed. Here already predicted MANET parameters like link lifetime, node lifetime, neighboring nodes and band width using bee colony optimization algorithm will be fuzzified. As a result of fuzzification, fuzzy rules will be formed to decide the node status. This information is made to exchange among all the nodes. Thus, the status of every node is verified before data transmission. Data will be transferred to any node if and only if the node status is strong. Else the data will be passed to some other node. With the simulated results, the minimized data loss and overhead will be obtained.
\end{abstract}

Keywords: MANET, fuzzification, evaluation rule, membership function, Defuzzification, BCO.

\section{Introduction}

Wireless network era paved way for many latest technologies in recent years. With the applications or wireless technology, many beneficial outcomes are there which made the world compact and easy to access everything. Mobile Ad-hoc Network (MANET) is one of the wireless network applications, contains nodesinmobility. Besides, MANET doesn't need any transmission media like base station or access point. This makes MANET an infrastructure less network. Fig 1 shows simple MANET with few nodes in it. 


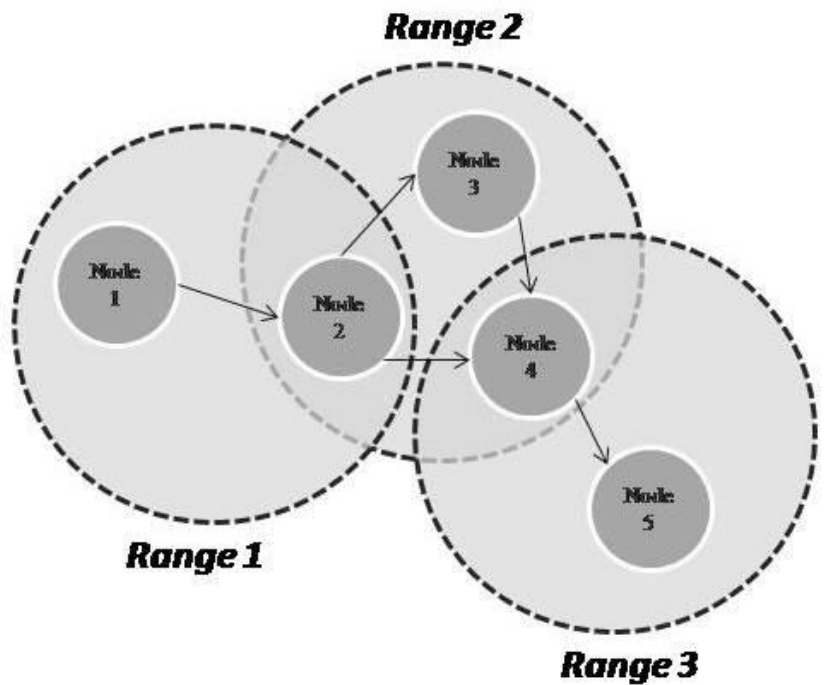

Fig 1Mobile Ad-hoc Network

Because of its mobility nature, MANET nodes are highly prone to link and route failure. When nodes travel from one place to another place link failure occurs. Whenever this happens, rerouting is needed to recover the link. Rerouting is not always possible if the network is very large and if rerouting is done in larger networks, control overhead will become a major problem. In order to avoid rerouting and overheads, an efficient routing mechanism has to be applied and intelligent routing should be done based on the predicted output from applied technology [1].

This paper deals with intelligent routing needs that are to be followed in MANET for efficient routing and to avoid larger reroutingoverhead. To choose best node to transmit packets, fuzzification is done on outputs obtained from bee colony optimization technique. As a result of fuzzification, rules will be framed to decide the status of node. With the decided output, data will be transmitted only if the node status is strong else it will be transmitted through some other node whose status is strong.

\section{Fuzzy Inference}

The process of convertingany crisp input into fuzzy output, obtaining some rules out of it and again converting it into another crisp output[3].For fuzzification and obtaining rules, some input has to be obtained. This input is given by applying bee colony optimization algorithmover MANET parameters in the first phase of this research.

\section{Bee Colony Optimization (BCO) algorithm}

Population based algorithms are very much effective for implementing any network based technologies. Bee Colony Optimization algorithm is also a population based algorithm where certain population of bees are involved in obtaining optimum solution. This algorithm is inspired by foraging behavior of natural bees. Foraging behavior is nothing but act of finding good honey source[6]. There involved 3 kinds of bees in this process.

i. Employee bee is responsible for searching forhoney. Once the honey source is found it will inform every other bee by dancing in the hive, the home of all bees. In the hive quality of honey source will be decided. 
ii. On looker bee will follow the employee bee whose honey source is decided as good one.

iii. Scout bee will abandon the poor quality honey source and corresponding employee bee will search some other source.

All the above natural process are applied in MANET by considering few of MANET parameters and some crisp value will be obtained for decision making about routing. These values are then fuzzified.

\section{RELATED WORK}

Protocols are set of rules formulated for connecting two kinds of devices over network. Protocol differs from wireless and wired networks. MANET is wireless network and there are 3 types of protocols in it[5].

\section{i. Table Driven or Proactive Protocols}

It is non-dynamic protocol where routing table is maintained for every node. With the help of table entries, routing is made. This is very good for smallest networks but not suitable for large networks where routing entries are to be maintained for large number of nodes.Certain Proactive Routing Protocols are DSDV, Wireless Routing Protocol (WRP), Global State Routing (GSR) and Cluster-head Gateway Switch Routing (CGSR) [5].

\section{ii. On demand or Reactive Protocols}

These are dynamic on demandprotocols. Here no routing table is maintained so it is really good for large network. But each and every node maintains a cache table where small amount of routing information is stored and used for near future. Reactive Protocols are Cluster Based Routing Protocol (CBRP), Dynamic Source Routing (DSR), TORA, Associativity-Based Routing (ABR), Signal Stability Routing (SSR) and Location Aided Routing (LAR) [5].

\section{iii. Hybrid Protocols}

Hybrid protocol combines both proactive and reactive protocols. While starting, it starts as proactive protocol and at the middle and at the finishing stage it converts into reactive protocols. Zone Based Routing Protocol (ZRP) is example for hybrid protocol [5]. Fig 2 shows MANET protocols. 


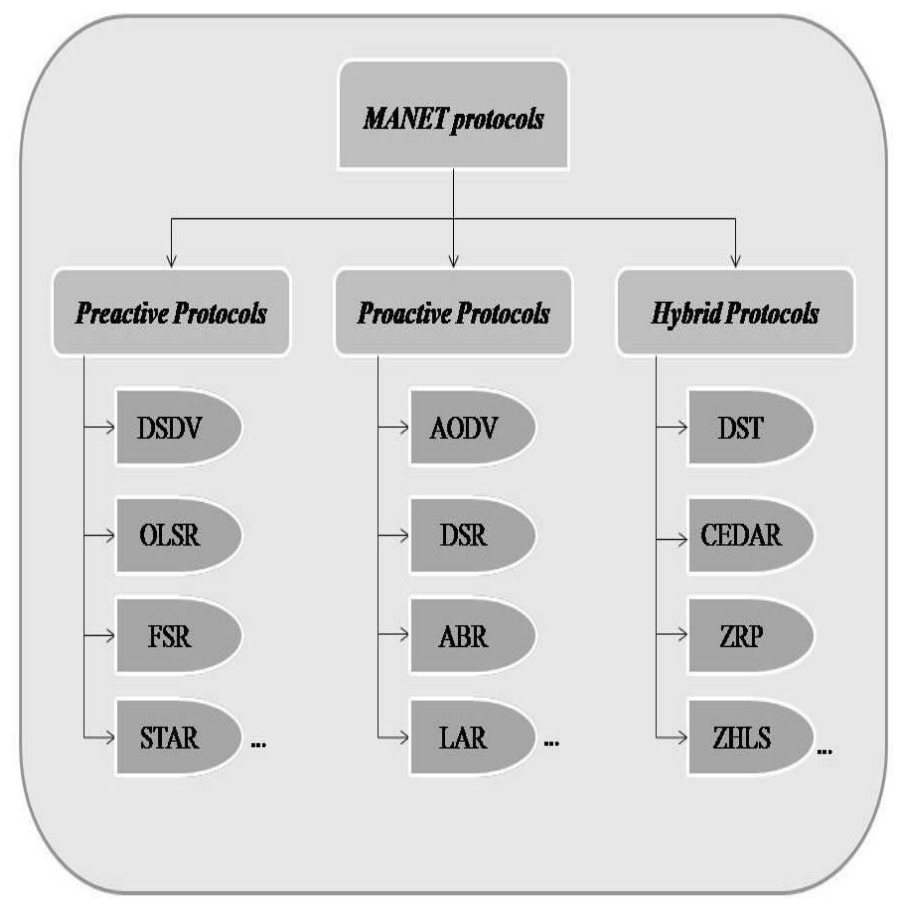

Fig 2 MANET Protocols

Since DSR is reactive protocol it suits better for implementing largest network like MANET. DSR does two jobs route discovery and route maintains. During route discovery, source sends Route Request (RREQ) packets to all nodes in the network. Nodes that are willing to grant route to particular packet will send Route Reply (RREP). Then source chooses one among the nodes for transmission. This occurs in route discovery. Route maintenance phase is responsible error checking in network. If any route fails or any error occurs along the path of the packet it sends route error packet to indicatethe occurrence of error. This protocol is chosen for research since it is efficient and dynamic in nature [5].

In a mobile ad hoc network (MANET) in which nodes move randomly within constrained areas. In this regard, the authors presented statistical models to accurately evaluate the distribution of the lifetime of a wireless linkIt was shown thata two-state Markov model is applied to compute link lifetime and further applied for the optimization of segmentation schemes of information stream. All these results are summarized and comprehensive analysis on throughput, delay, and storage requirements for MANETs with restricted node mobility are provided [7].

Devi et. al proposed particle swarm optimization (PSO)-based node and link lifetime prediction algorithm for routerecovery in MANET In this research Lifetime prediction algorithm using particle swarm optimization (PSO)-basedlifetime prediction algorithm for route recovery was proposed. This technique predicts the parameters like lifetime oflink and nodes. Using this prediction algorithm, the parameters are fuzzified and fuzzy rules have been formed to decide on the node status [2].

\section{Proposed Work}


All the above mentioned existing works have chosen only two parameters like link lifetime and node lifetime. Both the parameters are really important for efficient routing in MANET.But with only these parameters efficiency in routing cannot be obtained. So it is always wise to choose some more important MANET parameters for efficient routing.

In this research, available bandwidth, neighboring nodes along with link lifetime and node lifetime have been chosen for simulation. The first phase of this research applies BCO algorithm on MANET parameters like neighboring nodes, link lifetime, node lifetime and available bandwidthand depicts some crisp value for deciding node status. These crisp values are taken and fuzzifiedand optimum solution is obtained and discussed in this research paper. This is second phase of this research. The following are brief description of the MANET parameters considered for the research.

\section{i. $\quad$ Link Lifetime}

Link lifetime is the time period that the link is available continuously. It can be predicted by calculating the distance between two nodes over a particular period of time [7].

\section{ii. $\quad$ Node Lifetime}

Node lifetime represents the energy of node. There are two kinds of nodes one is active node and another one is inactive node. Active node drains energy faster than inactive node[7].

$$
\text { iii. Available Bandwidth }
$$

Bandwidth is a range of frequencies within a given band, in particular that used for transmitting a signal. Bandwidth can be depicted using link capacity and by considering rate of incoming and outgoing flows [7].

\section{iv. Neighboring Nodes}

Node with large number of neighboring nodes is best for transmission. Once a node fails, packets can be routed through some other neighboring node. So it is always good practice to take neighboring node as a parameter for efficient routing in MANET[7].

Fig 3 shows the work flow of fuzzy system. 


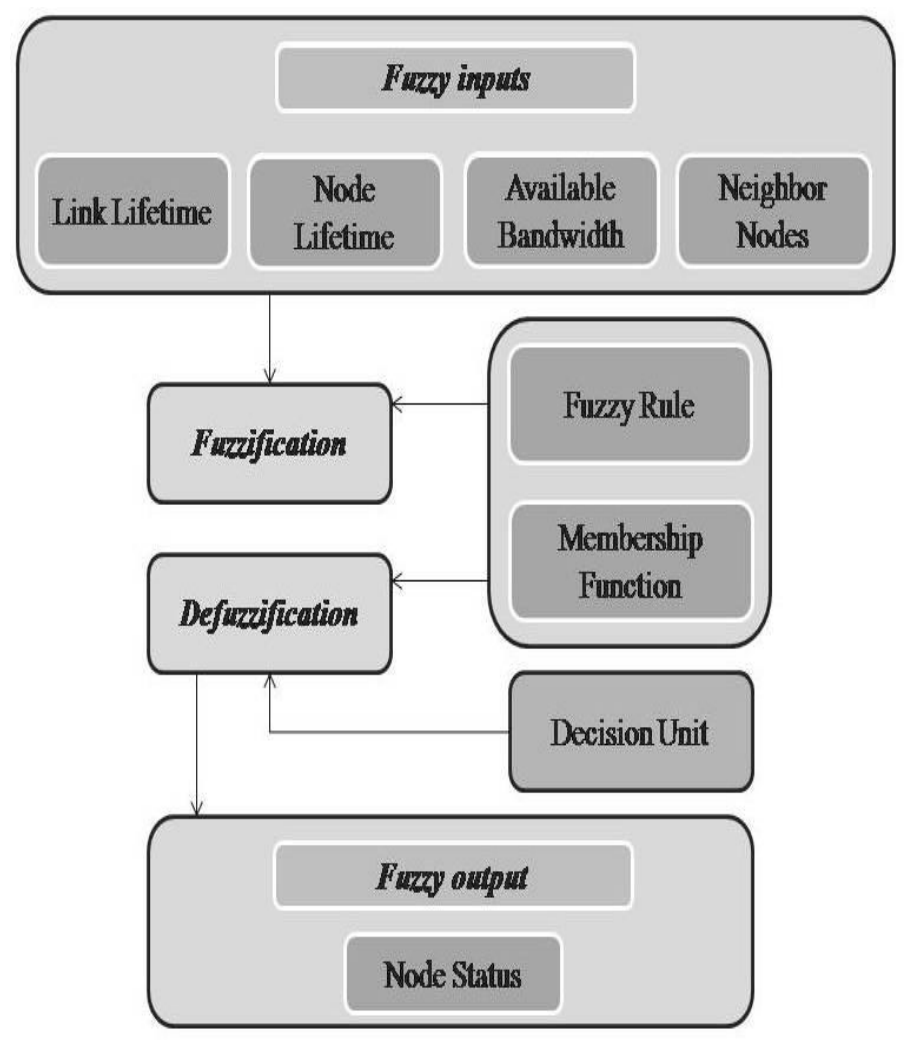

Fig 3 Fuzzy System model

Applying Fuzzy Inference on MANET Parameters

Fuzzy inference is process of taking some crisp input, obtaining some rules out of it and again converting the rules into some crispoutput. It consists of 3 majorfunctions
i. Fuzzification
ii. Rule Evaluation
iii. Defuzzification

The following are steps involved in fuzzy inference mechanism [3].

\section{Fuzzification}

1. Determining a set of fuzzy rules.

2. Fuzzifying the inputs using the input membership functions.

Rule Evaluation

1. Combining the fuzzified inputs according to the fuzzy rules to establish the rule strength.

2. Finding the consequence of the rule by combining the rule strength and the output membership function. 


\section{Defuzzification}

3. Defuzzifying the output distribute onwhenever a crisp outputis needed.

Outcome of all these information are needed for predicting status of MANET nodes. Fig4 shows basic functions of fuzzy inference system.

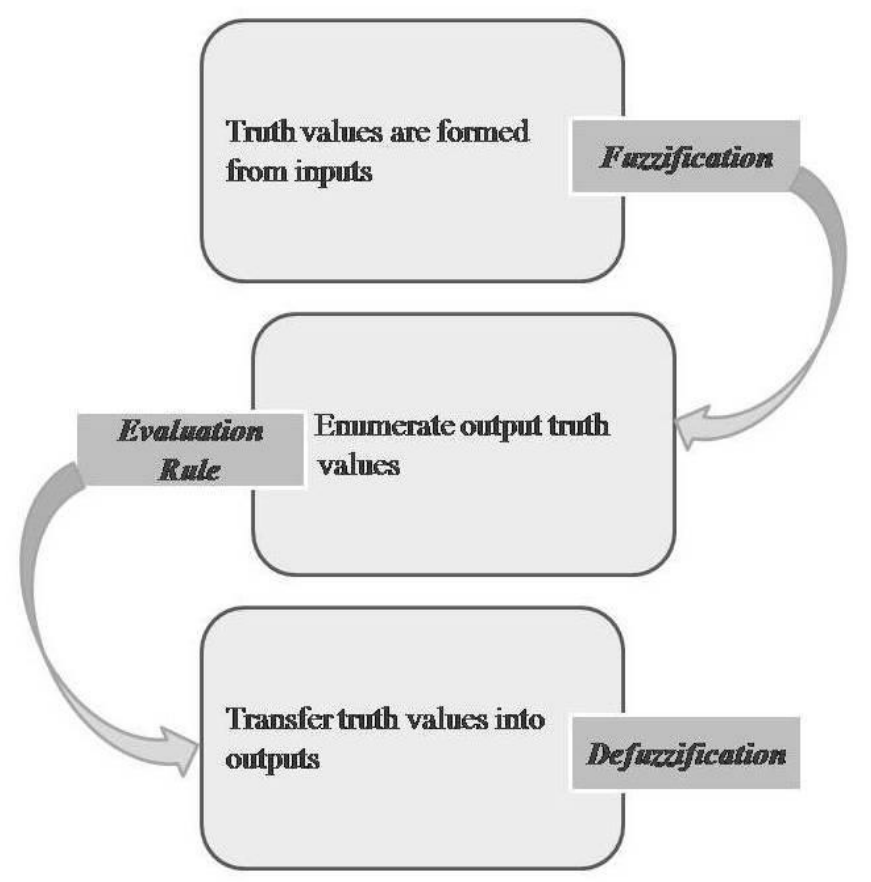

Fig 4 Fuzzy Inference System

\section{Fuzzification}

Fuzzificationis the process of transforming crisp values into fuzzy sets. Some membership functions are used to convert crisp input to fuzzy input. Here outcomes like link lifetime, node lifetime, bandwidth and neighboring nodes generated by BCO phase will be fuzzified. Fig5(a)(b)(c) shows membership functions of MANET parameters link lifetime, node lifetime, available bandwidth and neighboring nodes respectively. 


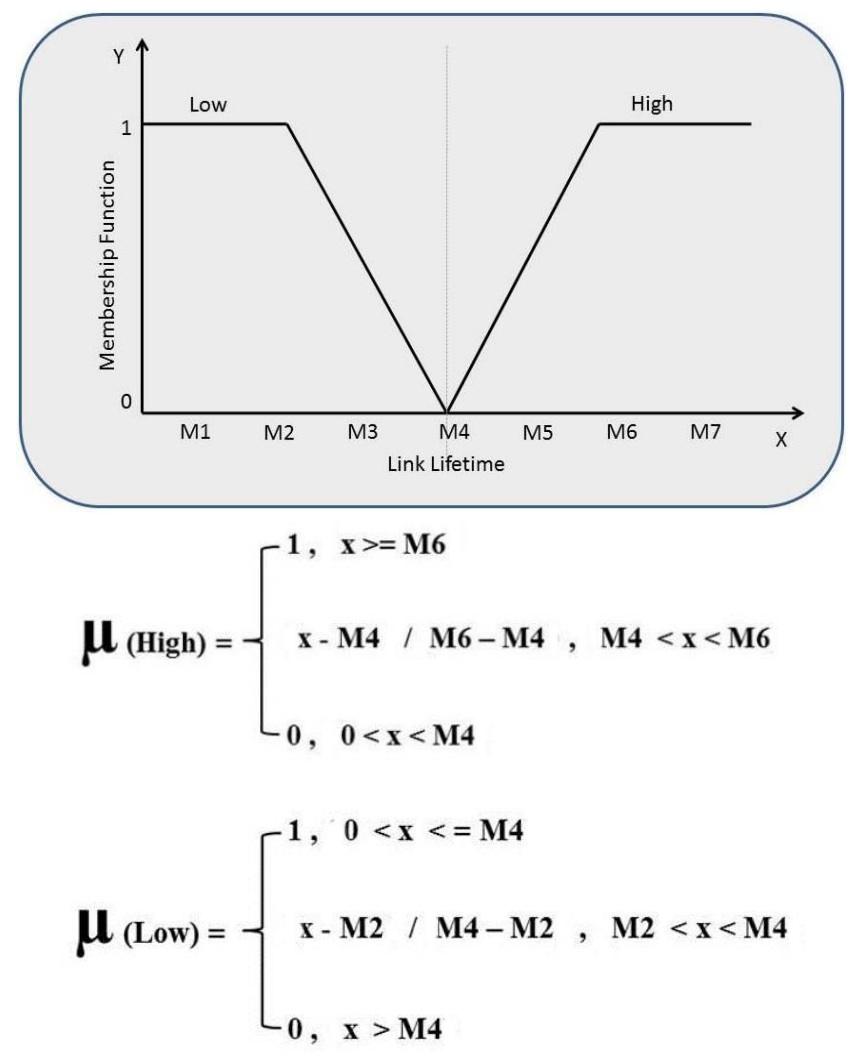

Fig 5 (a) Membership Function for Link Lifetime

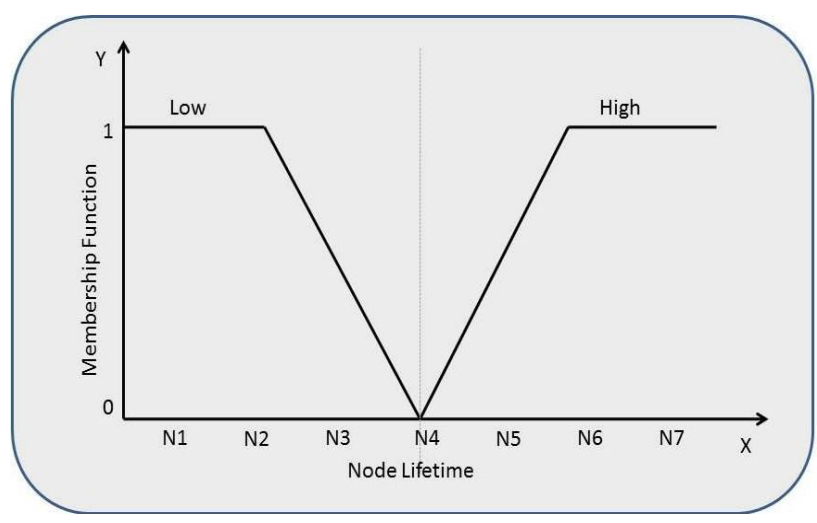




$$
\begin{aligned}
& \boldsymbol{\mu}_{(\text {High })}= \begin{cases}1, & \mathrm{x}>=\mathrm{N} 6 \\
\mathrm{x}-\mathrm{N} 4 / \mathrm{N} 6-\mathrm{N} 4, \mathrm{~N} 4<\mathrm{x}<\mathrm{N} 6 & \\
0, & 0<\mathrm{x}<\mathrm{N} 4\end{cases} \\
& \boldsymbol{\mu}_{(\text {Low })}= \begin{cases}1, & 0<\mathrm{x}<=\mathrm{N} 4 \\
\mathrm{x}-\mathrm{N} 2 / \mathrm{N} 4-\mathrm{N} 2, \mathrm{~N} 2<\mathrm{x}<\mathrm{N} 4 \\
0, & \mathrm{x}>\mathrm{N} 4\end{cases}
\end{aligned}
$$

Fig 5 (b) Membership Function for Node Lifetime

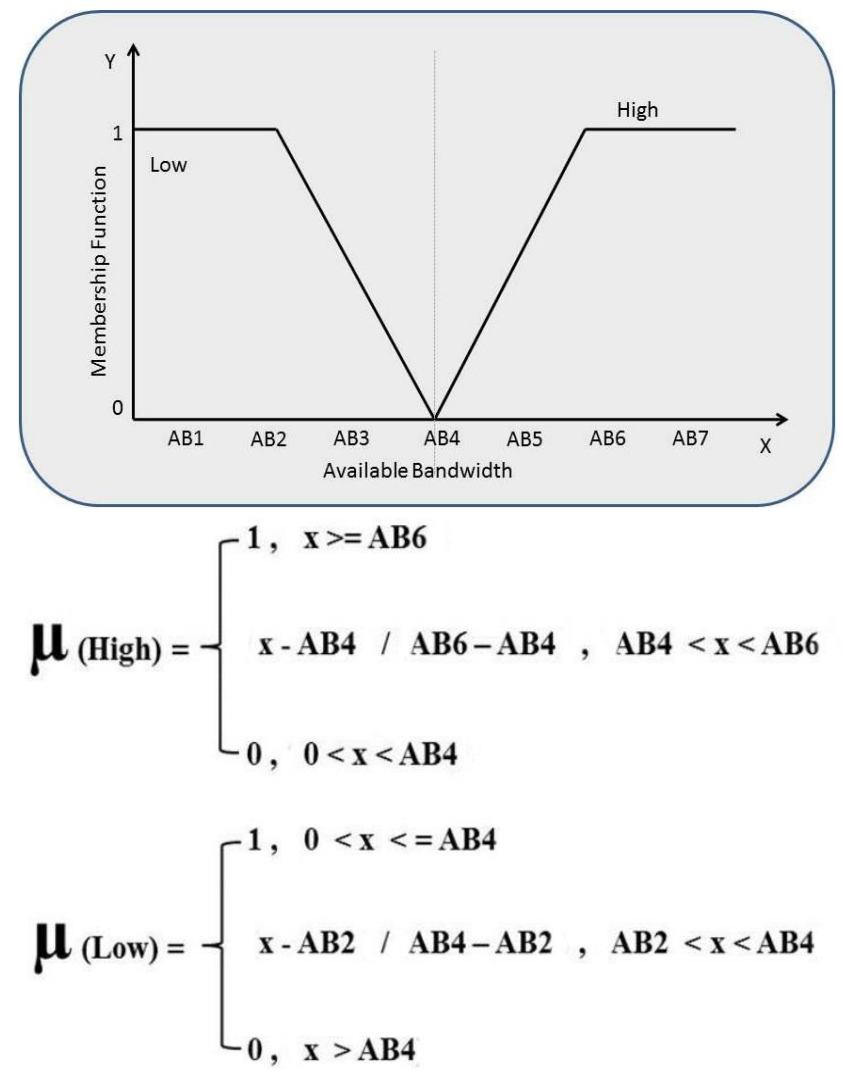

Fig 5 (c) Membership Function for Available Bandwidth 


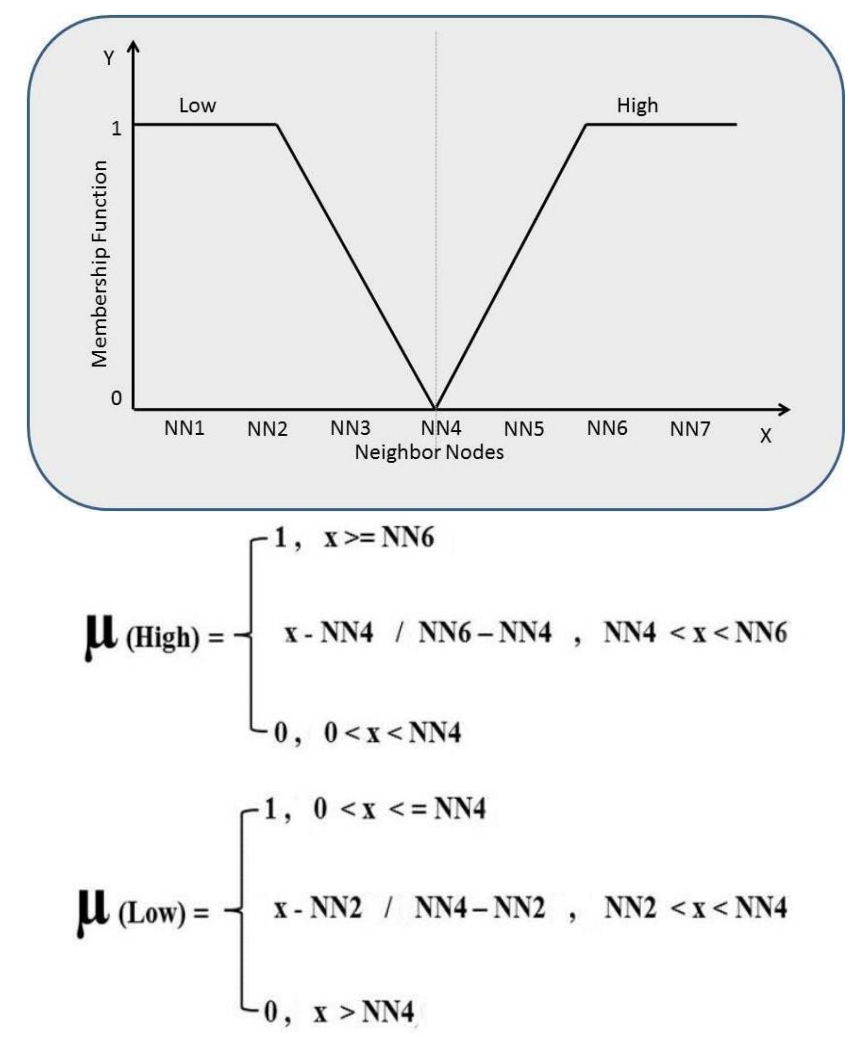

Fig 2.2 (d) Membership Function for Neighboring Nodes

\subsubsection{Rule Evaluation}

Fuzzy inputs are then converted into truth values and rules are framed for predicting node status. Table 2.1 shows sample fuzzy rules for determining node status. 


\begin{tabular}{|c|c|c|c|c|c|}
\hline Sno & $\begin{array}{c}\text { Link } \\
\text { Lifetime }\end{array}$ & $\begin{array}{c}\text { Node } \\
\text { Lifetime }\end{array}$ & $\begin{array}{c}\text { Available } \\
\text { Bandwidth }\end{array}$ & $\begin{array}{c}\text { Neighbor } \\
\text { Nodes }\end{array}$ & $\begin{array}{c}\text { Node } \\
\text { Status }\end{array}$ \\
\hline 1 & High & High & High & High & Strong \\
\hline 2 & High & High & High & Low & Strong \\
\hline 3 & High & High & Low & High & Strong \\
\hline 4 & High & High & Low & Low & Normal \\
\hline 5 & High & Low & High & High & Strong \\
\hline 6 & High & Low & High & Low & Normal \\
\hline 7 & High & Low & Low & High & Normal \\
\hline 8 & High & Low & Low & Low & Weak \\
\hline 9 & Low & High & High & High & Strong \\
\hline 10 & Low & High & High & Low & Normal \\
\hline 11 & Low & High & Low & High & Normal \\
\hline 12 & Low & High & Low & Low & Weak \\
\hline 13 & Low & Low & High & High & Normal \\
\hline 14 & Low & Low & High & Low & Weak \\
\hline 15 & Low & Low & Low & High & Weak \\
\hline 16 & Low & Low & Low & Low & Weak \\
\hline
\end{tabular}

Table 2.1 Fuzzy Rules for Determining Node Status

In the above table, based on the values of MANET parameters like link lifetime, node lifetime, bandwidth and neighboring nodes the node status is decided.

If link lifetime is high, node lifetime is low, bandwidth is low and neighboring node is high then the node status is "Normal".

If all the parameters are low, node status is "Weak".

If all are high, then the status will be "Strong".

Once a nodes' status is found next step is selecting that node for transmitting if and only if the status of node is strong or medium. Weak nodes will not be chosen for transmission and they will be eliminated. $\quad$ Fig6 shows the membership function for node status. 


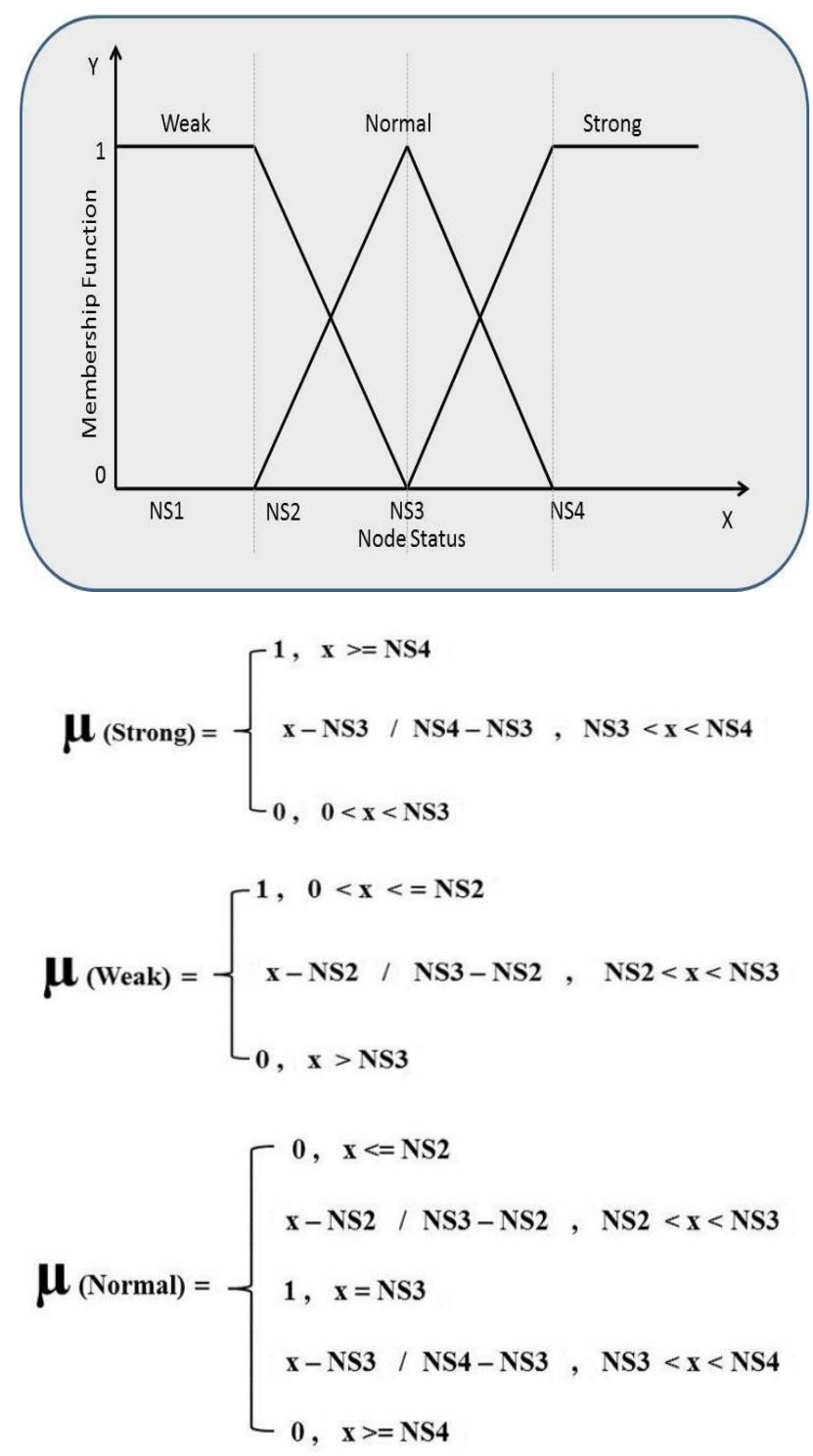

Fig 6 Membership Function for Node Status

\section{Defuzzification}

Defuzzification involves the process of transforming the fuzzy outputs to crisp outputs. The merged output of the fuzzy set is the input for the defuzzification process and a single crisp number is obtained as output. That is what called optimum solution.To obtain this, centroid defuzzification technique is used. This is known as centre of gravity or centre of area defuzzification which is more commonly used technique. This can be expressed as 


$$
\mathrm{x}^{*}=\frac{\sum_{\mathrm{i}=1}^{\mathrm{q}} \mathrm{z}_{\mathrm{i}} \mathrm{C}\left(\mathrm{z}_{\mathrm{i}}\right)}{\sum_{\mathrm{i}=1}^{\mathrm{q}}()}
$$

Where $\mathrm{x}^{*}$ is defuzzifier output, where, $\mathrm{q}$ is the number of sample values of the output, and $\mathrm{z}_{\mathrm{i}}$ is the value of the control output at the sample value.

Fig7 shows fuzzy based selection of nodes.

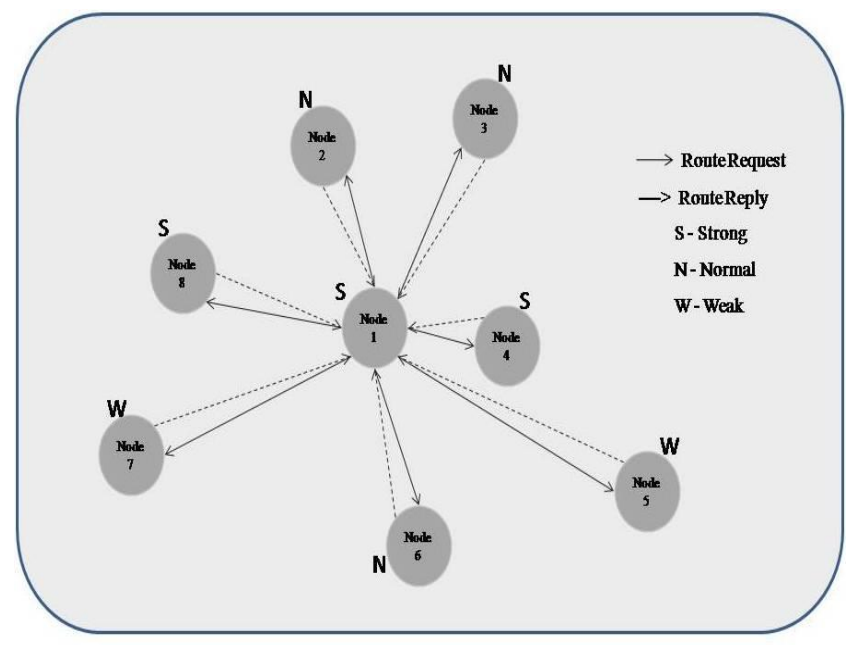

Fig 7 Fuzzy Based Selection of Nodes

\section{Simulation}

All the above metrics are simulated using Network Simulator 2.35 (NS-2.35). Network Simulator is an efficient tool for simulating real time network and obtaining results as obtained real time. NS-2.35 comes with NAM animator which animates the created network and shows it in GUI environment. With the help of NS-2.35 it is very easy to simulate very large network in single system. 


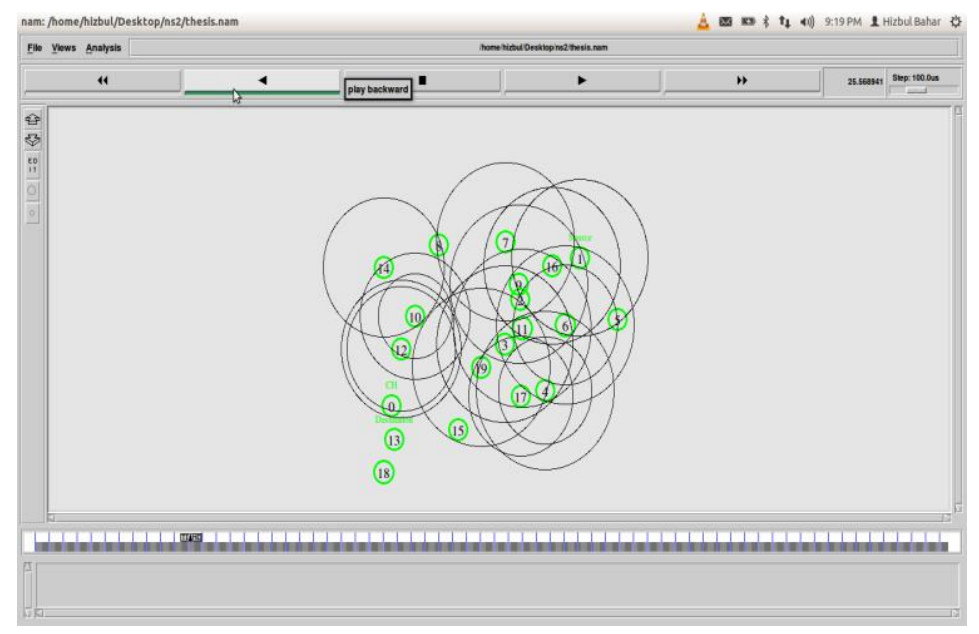

\section{Conclusion}

Fig 8 NAM window showing MANET nodes

MANET is mobile ad-hoc network comes with so many advantages. But there are much more disadvantages too. One of the disadvantages of MANET is route failure due to mobility nature of MANET nodes. This drawback is analysed in this paper and solutions are discussed in detail. With the help of BCO algorithm crisp values can be obtained and then they can be fuzzified to get the optimum solution. Thus this paper presents a solution which can highly minimize one of the MANET's drawbacks and enhance efficiency in routing.

\section{Reference}

[1] S. S. Tyagi “Study of MANET: Characteristics, Challenges, Application and Security Attacks." Int. J. adv. Res. Comput. Sci. Softw. $\operatorname{Eng}(2013)$

[2] Devi Manickavelu and RhymendUthariarajVaidyanathan. "Particle swarm optimization (PSO)based nodeand link lifetime prediction algorithm for routerecovery in MANET." EURASIP. Jo Wireless Com\&nw.(2014)

[3] Serge Guillaume "Designing Fuzzy Inference Systems from Data: An Interpretability-Oriented Review.” IEEE TRANSACTIONS ON FUZZY SYSTEMS, VOL. 9, NO. 3 (2001)

[4]E. Hemalatha Jai Kumari and Dr. Kannammal"Dynamic Shortest Path Routing In Mobile Adhoc Networks Using Modified Artificial Bee Colony Optimization Algorithm.” Int. J. adv. Res. Comput.Sci.\& Info. Tech. (2014)

[5] MehranAbolhasan ,TadeuszWysockiErykDutkiewicz"A review of routing protocols for mobile ad hoc networks"

[6] Dušan Teodorović1, Mauro Dell Orco "Bee Colony Optimization - A Cooperative Learning Approach to Complex Transportation Problems."

[7] Xianren Wu1, Hamid R. Sadjadpour and J.J. Garcia-Luna-Aceves "Link Lifetime as a Function of Node Mobility in MANETs with Restricted Mobility: Modeling and Applications." 